\title{
Nucleation kinetics and dielectric characterization of L-Proline Potassium Chloride (LPPC) single crystals
}

\author{
V. Vasantha Kumari ${ }^{1}$, P. Selvarajan ${ }^{2}$ \\ ${ }^{l}$ Govindammal Aditanar College for Women, Tiruchendur, Tamil Nadu, India \\ ${ }^{2}$ Aditanar College of Arts and Science, Tiruchendur, Tamil Nadu, India
}

\begin{abstract}
Semiorganic single crystals of L-proline potassium chloride (LPPC) were successfully grown from its aqueous solution by slow evaporation solution growth method at room temperature. The nucleation studies were carried out for the L-proline potassium chloride salt at room temperature. The critical nucleation parameters such as radius of critical nucleus, Gibbs' free energy change, and number of molecules in the critical nucleus were determined. Dielectric measurements for the grown L-proline potassium chloride crystals were carried out for different frequencies such as $1 \mathrm{kHz}, 10 \mathrm{kHz}, 100 \mathrm{kHz}, 1 \mathrm{MHz}$, and different temperatures ranging from $30^{\circ}$ to $120^{\circ} \mathrm{C}$. It is found that the dielectric constant and dielectric loss increases with increase of temperature and decreases with increase of frequency whereas a.c conductivity increases with increase in temperature and frequency.
\end{abstract}

Keywords: ac conductivity, critical radius, Dielectric constant, Dielectric loss, Nucleation

\section{Introduction}

Proline is an abundant amino acid in collagen and is exceptional among the amino acids because it is the only one in which the amino group is a part of pyrrolidine ring, making it rigid and directional in biological systems [1]. Single crystals of L-proline show no centre of symmetry and their NLO coefficients have been examined by Booma Devi and Dhanasekaran [2]. Some of the L-proline based materials such as prolinium picrate [3], L-proline cadmium chloride monohydrate [4], prolinium tartarate [5], dichlorobis L-proline (zinc II) [6], L-proline lithium chloride monohydrate [7], etc. have been reported. In the present work, concentration towards dielectric studies is given, since dielectric measurement is one of the useful characterization of electrical response of crystals. Studies of the dielectric properties of crystals give information about the electric field distribution within the solid. The frequency dependence of these properties gives a great insight into the materials applications. The different polarization mechanisms in crystals can be understood from the study of dielectric constants as a function of frequency and temperature.

\section{Nucleation Studies}

Nucleation kinetic studies have been carried out by measuring the induction period which is defined as the amount of time elapsed between the achievement of a supersaturated solution and the observation of nuclei. Experimentally the induction period was measured by isothermal method and it was used to evaluate the interfacial tension, Gibbs free energy change, critical radius and other nucleation parameters. The apparatus used for induction period is a constant temperature bath (controlled to an accuracy of $\pm 0.01{ }^{\circ} \mathrm{C}$ ) and a glass beaker. The glass beaker is used as a nucleation cell. Using the solubility data, the supersaturated aqueous solution of the sample in the corning glass beaker was prepared, preheated slightly and it was stirred continuously for about 2 hours using a magnetic stirrer to ensure the homogeneous concentration. The nucleation cell was then loaded into a constant temperature bath and illuminated using a powerful lamp to observe the formation of a nucleus. The time period that passes between the attainment of supersaturation and appearance of the first visible speck in the supersaturated solution is the induction period $(\tau)$. The induction period measurements were performed at different supersaturation ratios (S) at room temperature $\left(30{ }^{\circ} \mathrm{C}\right)$. The variation of induction period with the supersaturation ratio for the sample is shown in the Fig. 1 . It is observed that the induction period decreases with increase of supersaturation ratio. The interfacial tension is calculated as 2.8145 milli joules $/ \mathrm{m}^{2}$. Using the interfacial tension value, the nucleation parameters such as the radius of the critical nucleus $\left(r^{*}\right)$, the free energy for the formation of the critical nucleus $\left(\Delta \mathrm{G}^{*}\right)$ and the number of molecules in the critical nucleus $\left(i^{*}\right)$ have been calculated for the controlled nucleation condition and the results are given in the table 1. From the results it is noticed that the nucleation parameters such as radius of critical nucleus, Gibbs' free energy change, and number of molecules in the critical nucleus decrease with supersaturation ratio. The variables that affect the nucleation rate are $\mathrm{pH}$, supersaturation, temperature and interfacial tension of the solution. The calculated critical radius $\mathrm{r}^{*}$ decreases with the increase of supersaturation and consequently the 
nucleation rate $(\mathrm{J})$ increases with increase of supersaturation. The nucleation rate is observed to be increasing with thesupersaturation ratio and if the nucleation rate is low, the formation of multi-nuclei in the solution will be less and hence big-sized crystals could be grown [8-15].

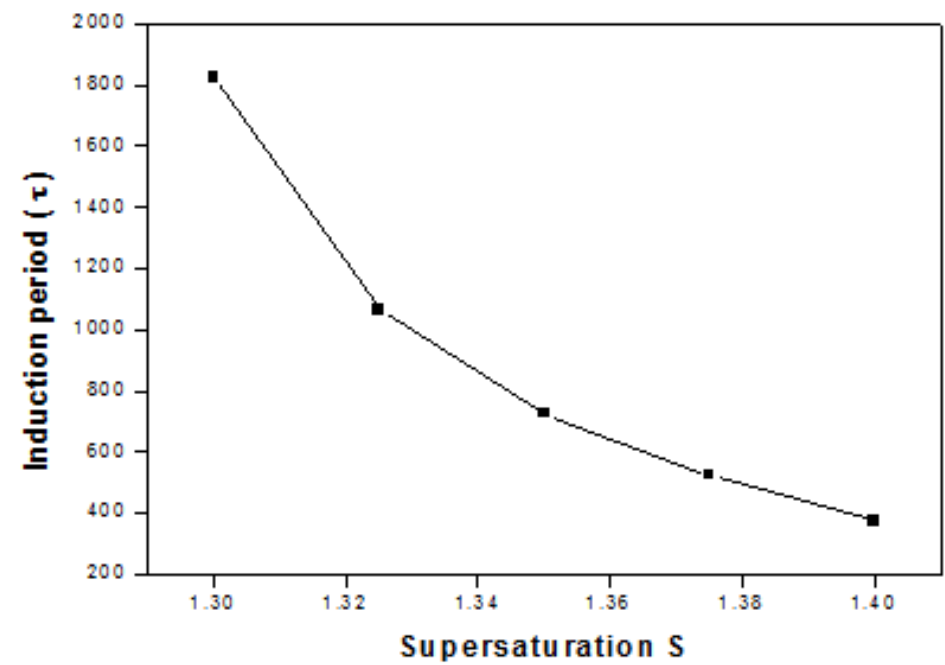

Figure 1: Variation of induction period with supersaturation ratio for L-proline potassium chloride salt

Table 1 Critical nucleation parameters of LPPC crystal

\begin{tabular}{|c|c|c|c|c|c|}
\hline S.No. & $\mathrm{S}=\mathrm{C} / \mathrm{Co}$ & $\mathrm{G}^{*} \times 10^{-21} \mathrm{~J}$ & J (x10 ${ }^{28}$ nuclei/sec/vol.) & $\mathrm{r}^{*} \times 10^{-10} \mathrm{~m}$ & $\mathbf{i}^{*}$ \\
\hline 1 & 1.3 & 4.91 & 30.78 & 6.45 & 20 \\
\hline 2 & 1.325 & 4.26 & 35.98 & 6.01 & 16 \\
\hline 3 & 1.35 & 3.75 & 40.69 & 5.64 & 13 \\
\hline 4 & 1.375 & 3.33 & 44.90 & 5.32 & 10 \\
\hline 5 & 1.4 & 2.98 & 48.83 & 5.03 & 8 \\
\hline
\end{tabular}

\section{Growth of LPPC crystals}

Single crystals of LPPC were grown from their aqueous solution using slow solvent evaporation technique. The supersaturated solution was prepared in accordance with the solubility data. The solution was stirred at 50oC using a temperature controlled magnetic stirrer for three hours to obtain a homogeneous solution. The solution was filtered using Whatmann filter paper and the filtered solution was transferred into a beaker and closed with perforated sheet. The solvent was allowed to evaporate and numerous tiny crystals were formed at the bottom of the container due to spontaneous nucleation. Good optical quality crystals of were harvested after a period of 15 days. The photograph of the as grown LPPC crystal is shown in Fig. 2 and the grown crystals are found to be stable, transparent and colorless with well-defined crystal faces and edges.

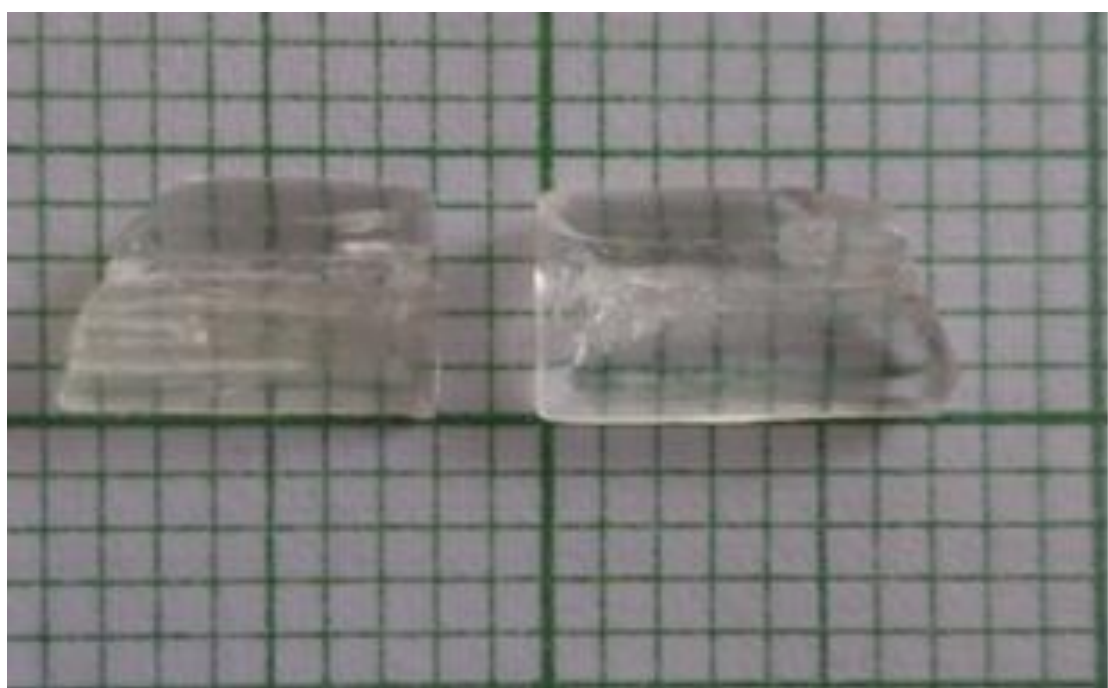

Figure 2: Photograph of LPPC crystals 


\section{Dielectric response of LPPC crystal}

Dielectric properties are correlated with electro-optic property of the crystals [6]. The polished single crystal of LPPC was used for dielectric studies. The sample was electroded on either side with graphite coating to make it behave like a parallel plate capacitor. Using an Agilent LCR meter, the capacitance and dielectric loss $(\tan \delta$ ) were measured at the frequency of $100 \mathrm{~Hz}, 1 \mathrm{kHz}, 10 \mathrm{kHz}$ and $100 \mathrm{kHz}$ and $1 \mathrm{MHz}$ at various temperatures. Repeated trials were performed to ascertain the correctness of the observed results. The dielectric constant of the crystal was calculated using the relation $\epsilon_{\mathrm{r}}=\mathrm{C} / \mathrm{C}_{\mathrm{o}}$, where $\mathrm{C}$ is the capacitance of the capacitor in the presence of the crystal and $\mathrm{C}_{\mathrm{o}}$ is the capacitance in air. The measurement of dielectric constant and loss as a function of frequency and temperature gives the ideas of electrical processes that are taking place in materials. The values of dielectric constant and loss factor were measured using an LCR meter at different frequencies and temperatures. The dielectric constant and dielectric loss is found to decrease with increase in frequency as shown in figures 3 and 4 . The high values of $\epsilon_{\mathrm{r}}$ at low frequencies may be due to presence of all the four polarizations namely, space charge, orientational, ionic and electronic polarizations and its low value at high frequencies may be due to the loss of significance of these polarizations gradually [16 - 18]. In accordance with Miller rule, the lower value of dielectric constant at higher frequencies is a suitable parameter for the enhancement of SHG coefficient [19]. The low value of dielectric loss at high frequency reveals the high optical quality of the crystal with lesser defects, which is the desirable property for NLO applications [20]. The dielectric constant of dispersive medium decreases because the term contributing todielectric constant from iondipole interactions is compensated by the thermal energy leading to the relaxation of polarization [21]. From the results, it is observed that as the temperature increases, the value of dielectric constant and dielectric loss increase to a considerable value as shown in figures 5 and 6.

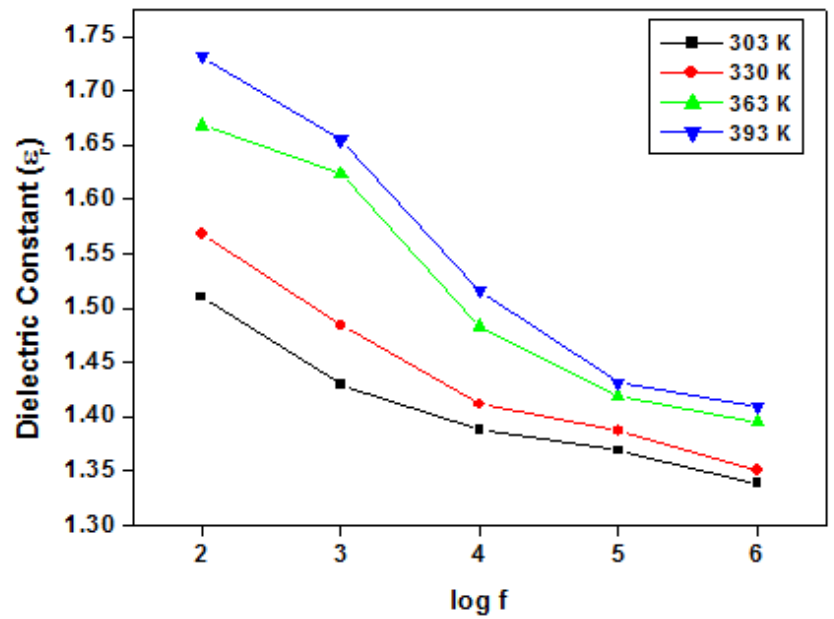

Figure 3: Plot of dielectric constant versus log f for LPPC crystal

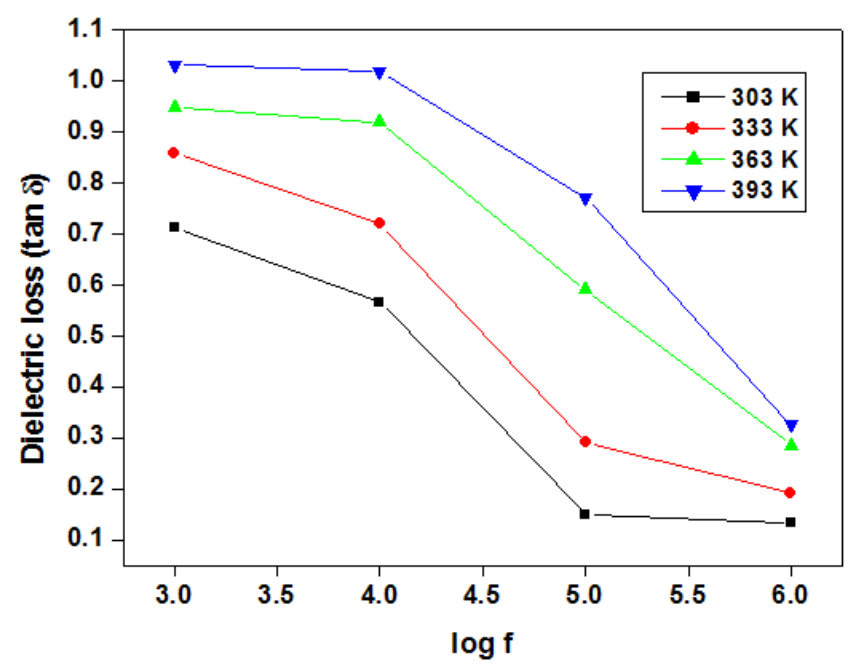

Figure 4: Plot of dielectric loss versus log f for LPPC crystal 


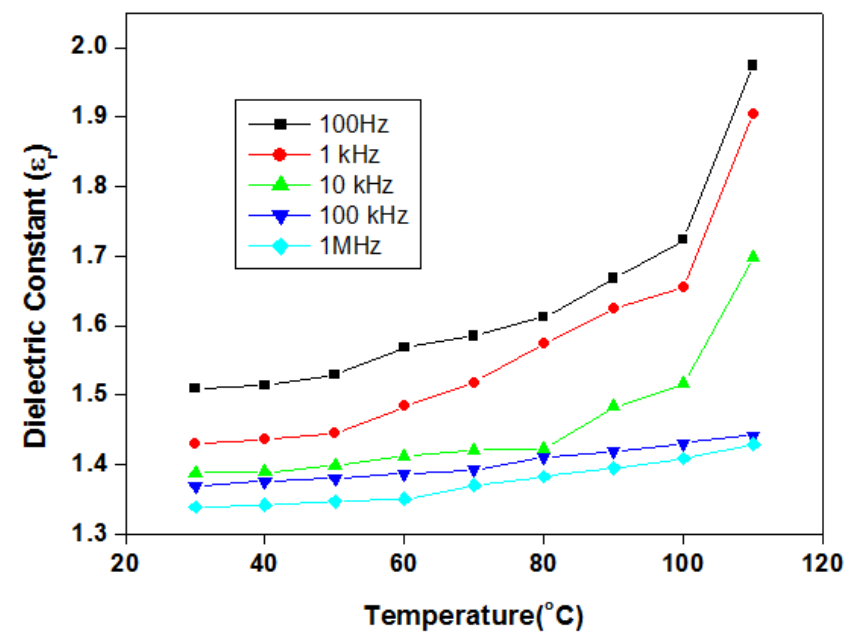

Figure5: Plot of dielectric constant versus temperature for LPPC crystal

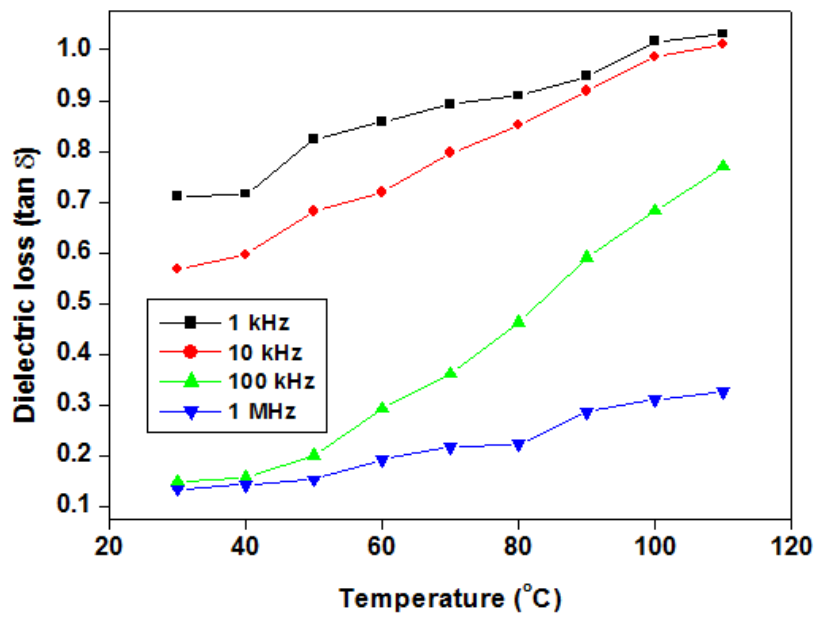

Figure 6: Plot of dielectric loss versus temperature for LPPC crystal

The ac conductivity was calculated at different frequencies using the formula. Figure 7 shows the plot of ac conductivity versus 1000/T. It is evident from the graph that the conductivity increases with temperature. The Arrhenius plot of $\ln \sigma_{\mathrm{ac}}$ versus 1000/T is shown in figure 8. The activation energy for ionic migration was estimated from the graph. The line of best fit for the plot of $\ln \sigma_{\text {ac }}$ versus 1000/T obeys Arrhenius relationship. The sample exhibits Arrhenius type conductivity behavior and the activation energy of LPPC crystal is found to be $0.911 \mathrm{eV}$.

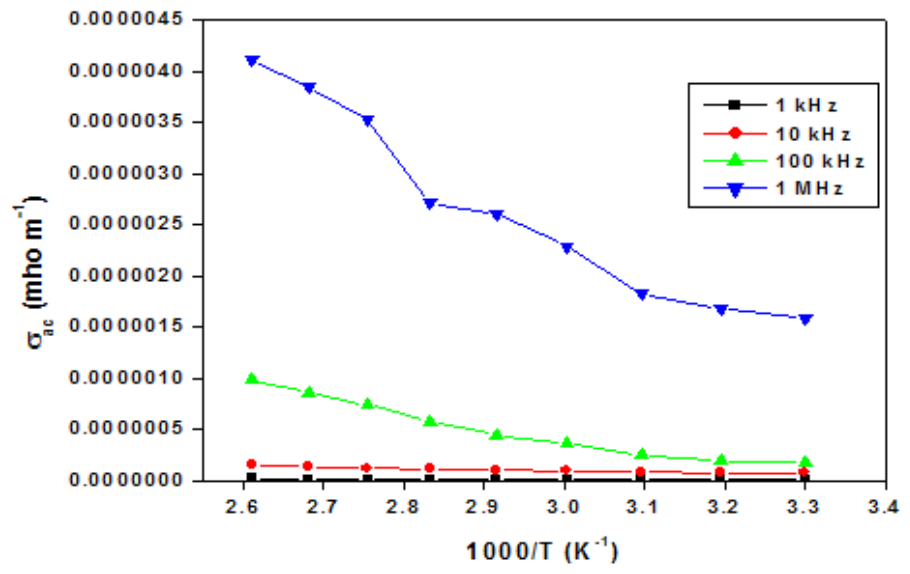

Figure 7: Plot of $\sigma_{\mathrm{ac}}$ versus 1000/T 


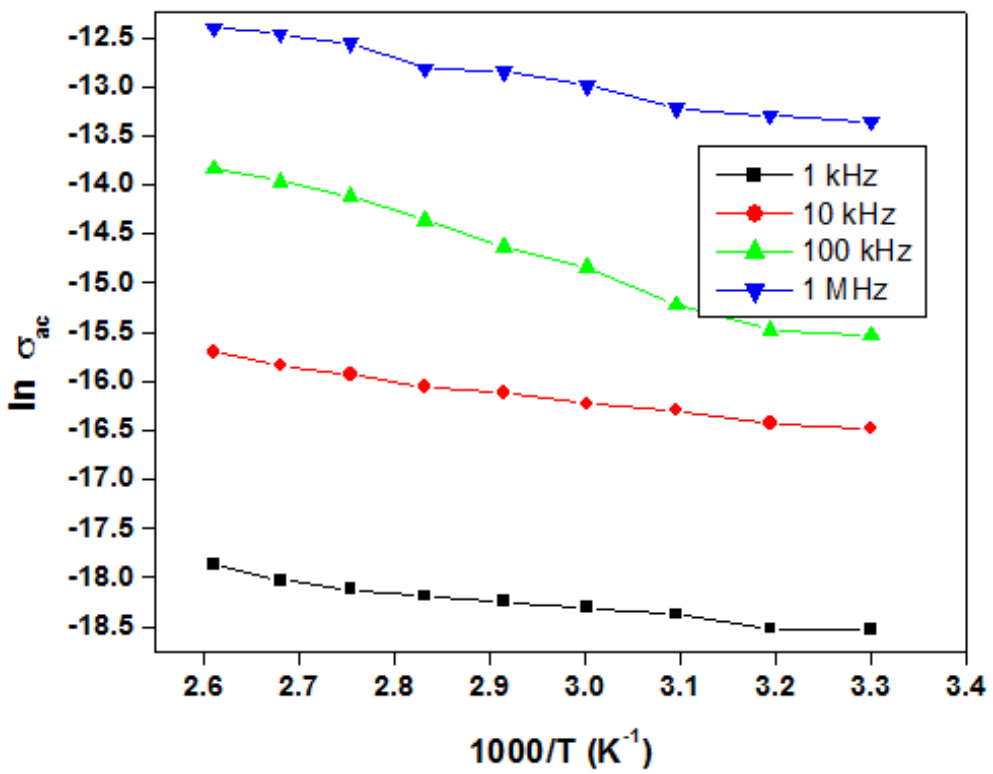

Figure 8: Plot of $\ln \sigma_{\mathrm{ac}}$ versus 1000/T

\section{Conclusion}

Optical quality single crystals of L-proline potassium chloride (LLPC) were grown using solution growth technique. The nucleation studies were carried out for the L-proline potassium chloride salt at room temperature and the critical nucleation parameters such as radius of critical nucleus, Gibbs' free energy change, and number of molecules in the critical nucleus were determined. Dielectric measurements for the grown Lproline potassium chloride crystals were carried out for different frequencies such as $1 \mathrm{kHz}, 10 \mathrm{kHz}, 100 \mathrm{kHz}, 1$ $\mathrm{MHz}$, and different temperatures ranging from $30^{\circ}$ to $120^{\circ} \mathrm{C}$. It is found that the dielectric constant and dielectric loss increases with increase of temperature and decreases with increase of frequency whereas a.c conductivity increases with increase in temperature and frequency. The activation energy of LPPC crystal is found to be $0.911 \mathrm{eV}$.

\section{Acknowledgements}

The authors would like to thank the staff members of PSN college of Technology, Melathediyoor for helping us to carry out the dielectric characterization. Also we thank the authorities of the management of Aditanar College of Arts and Science, Tiruchendur, Govindammal College for Women, Tiruchendur and S.T. Hindu College, Nagercoil for the encouragement given to us to carry out the research work.

\section{References}

[1]. S. Myung, Pink, M.H. Baik and David Clemmer,DL-Proline, ActaCrystallography C, 61, 2005, 506-508.

[2]. S. Boomadevi and R.Dhanasekaran, Synthesis, crystal growth and characterization of L-pyrrolidone -2 -carboxylic acid (L-PCA) crystal, J. Crystal Growth, 261,2004, 70-76.

[3]. T. Uma Devi., N. Lawrence, R. Ramesh Babu and K.Ramamurthi, Growth and Characterization of L-prolinium picrate single crystal: A promising NLO crystal, J. Crystal Growth, 310,2008, 116-123.

[4]. J. Thomas Joseph Prakash and S. Kumararaman, Growth and characterization of L- proline cadmium chloridemonohydrate single crystals, Materials Letters, 62, 2008 4097-4099.

[5]. S.Suresh, A. Ramanand, D.Jayaraman and P.Mani, Dielectric studies of L-Prolinium Tartrate (LPT) NLO Single crystals, Journal of optoelectronics and Advanced materials, 4,2010, 1743-1746.

[6]. G. Anandha Babu and P.Ramasamy, Synthesis, crystal growth and characterization of novel semiorganic nonlinear optical crystal: Dichlorobis (1-proline) zinc (II), Materials Chemistry and Physics, 113,2009, 727-733.

[7]. T.UmaDevi, N.Lawrence, R.Ramesh Babu, S. Selvanayagam, Helen Stoeckli- Evans and K. Ramamurthi, Synthesis, Crystal Growth and Characterization of L-Proline Lithium Chloride Monohydrate: A New Semiorganic Nonlinear Optical Material,Cryst. Growth Des., 9(3), 2009, 1370-1374.

[8]. N.P. Zaitseva, L.N. Rashkovich and S.V. Bagatyacva, Application of solution techniques to rapid growth of organic crystals, J. Cryst. Growth, 148, 1995,276-278.

[9]. A.E. Nielsen and S. Sarig, Kinetics of precipitation, J. Cryst. Growth, 8, 1971, 13-16.

[10]. N.P. Rajesh, V. Kannan, P.S. Raghavan, P. Ramasamy and C.W. Lan, Nucleation studies and crystal growth of $\mathrm{NH}_{4} \mathrm{H}_{2} \mathrm{PO}_{4}$ doped with thiourea in supersaturated aqueous solutions, Mater. Chem. Phys, 76, 2002, 181-186. 
[11]. S. Sathishkumar, T. Balakrishnan, K. Ramamurthi and S. Thamotharan, Spectrochimica Acta Part A: Molecular and Biomolecular Spectroscopy, 138 (2015) 187-195.

[12]. A.C. Zattlemoyer, Nucleation(New York,Dekker1969), pp. 241.

[13]. S.Boomadevi, R. Dhanasekaran and P. Ramasamy, Investigations on nucleation and growth kinetics of urea crystals, Cryst. Res, Technol., 37, 2002, 159-168.

[14]. F. Joseph Kumar, D. Jayaraman, C. Subramaniam and P. Ramasamy, Nucleation kinetic study of KTiOPO crystallising from high temperature solutions, J. Cryst.Growth, 137, 1994, 535-537.

[15]. N.P. Rajesh, V. Kannan, P. Santhana Raghavan, P. Ramasamy and C.W. Lan, Nucleation Studies and Crystal growth in super saturated aqueous solution of (NH4)H2P04 doped with thiourea, Materials Chemistry and Physics, 76, 2002, $181-186$

[16]. K.V. Rao and A. Samakula, Dielectric Properties of Cobalt Oxide, Nickel Oxide, and Their Mixed Crystals, J. Appl. Phys., 36, 1965, 2031-2038.

[17]. P. Selvarajan, B.N. Das, H.B. Gon and K.V. Rao, Investigation on the growth and properties of (LA)X(K2SO4)1-X single crystals, J. Mat. Sci., 29, 1994, 4061.

[18]. N.V. Prasad, G. Prasad, T. Bhimasankaran, S.V. Suryanarayana and G.S. Kumar,Dielectric properties of cobalt doped cadmium oxalate crystals, Indian J. Pure Appl. Phys., 34,1996, 639- 643.

[19]. U. Von Hundelshausen, Electropic Effect and Dielectric Properties of Cadmium - Mercury -Thiocyanate crystal,Phys. Lett., 34 A(7), 1971, 405-406.

[20]. C. Balarew and R. Duhlew, Application of the Hard and Soft Acids and Bases Concept to Explain Ligand Coordination in Double Salt Structures,J. Solid State Chem., 55, 1984, 1-6.

[21]. C.P. Smyth, Dielectric Behavior and Structure,(New York, McGraw-Hill, 1955). 